
et dans les oeuvres de ses contemporains

\title{
Justice et violence dans les dénouements tragiques de Shakespeare
}

\section{Pierre Spriet}

Marie-Thérèse Jones-Davies (éd.)

\section{(2) OpenEdition}

\section{Journals}

Édition électronique

URL : http://journals.openedition.org/shakespeare/122

DOI : $10.4000 /$ shakespeare.122

ISSN : 2271-6424

Éditeur

Société Française Shakespeare

Édition imprimée

Date de publication : 1 novembre 1980

Pagination : 71-84

Référence électronique

Pierre Spriet, « Justice et violence dans les dénouements tragiques de Shakespeare », Actes des congrès de la Société française Shakespeare [En ligne], 2 | 1980, mis en ligne le 01 novembre 2007, consulté le 30 avril 2019. URL : http://journals.openedition.org/shakespeare/122 ; DOI : 10.4000/ shakespeare.122 


\section{SOCIÉTÉ FRANC̣AISE SHAKESPEARE}

\section{ACTES DU CONGRĖS 1980}

DIRECTEUR DE LA PUBLICATION

M.T. Jones - Davies

JEAN TOUZOT Libraire - Editeur 38 , rue Saint-Sulpice 75278 PARIS CEDEX 061981 


\section{JUSTICE ET VIOLENCE DANS LES DENOUEMENTS TRAGIQUES DE SHAKESPEARE}

Prétendre que le théâtre de Shakespeare est uniformément moral, ce serait se condamner à n'en faire qu'une lecture partiale et partielle. Il n'apparaît pas possible d'échapper au scandale du mal et de l'injustice, sauf dans les comédies qui, par définition, euphémisent ce mal en en refusant les conséquences. Lire, avec une partie de la critique traditionnelle, dans les tragédies la multiple illustration de la nocivité de la passion, c'est évacuer le scandale mais aussi la tragédie.

On a tort de vouloir rapprocher Shakespeare d'un Sidney, d'un Spenser ou d'un Ben Jonson. Tout les oppose. Le premier n'est pas et ne veut pas être un homme de lettres; c'est un amuseur qui se propose d'attirer dans son théâtre un public nombreux. Les autres sont des littérateurs qui, conformément à l'idée que toute la Renaissance se fait de l'art, entendent donner à leur œuvre une perspective didactique et morale.Sidneyveut faire de son Arcadia le grand poème héroïque, romantique, philosophique et chrétien que l'Angleterre de la Renaissances se doit de posséder. Il écrit pour manifester la vérité et il ne conçoit pas que son cuvre puisse ne pas exalter cette vérité chrétienne qui est pour lui la vérité tout court ${ }^{1}$. Il importe peu de savoir si Shakespeare s'oppose à ces vues, mais il est évident qu'il n'entend pas en faire la substance de son théâtre : il n'appartient pas au monde des lettres et il ne se préoccupe pas de manifester la vérité mais de servir son public en lui offrant les histoires qui lui plaisent. Le théâtre de Shakespeare ne prouve rien; il représente. Il n'est didactique que dans la mesure où tout spectacle qui met en scène des hommes en situation de communication, de coopération, de tension ou de conflit révèle au public des aspects de lui-même qu'il connaissait mal et contribue ainsi à structurer la vision même qu'il se fait du réel. Ce qui caractérise le théâtre de Shakespeare par opposition à l'œuvre écrite de ses contemporains du monde des lettres, c'est l'absence d'une référence idéologique unificatrice. Il ne refuse pas le platonisme moral d'un Sidney ni plus généralement le stoïcisme christianisé de nombre de contemporains, mais ce ne sont pas ces systèmes qui structu- 
rent ses pièces. Il ne célèbre ni un ordre métaphysique ni un ordre moral ni - en dehors des pièces historiques - un ordre politique. Ses re-présentations ne sont pas au service d'une idéologie, ce qui veut pas dire qu'elles n'expriment pas des idéologies mais que le dramaturge ne les reprend pas à son compte : il est la multiple voix de ses personnages, non un prophète qui dit le droit.

Dans le théâtre de Shakespeare, il n'y a que des relations. La scène s'installe délibérément à ce niveau interrelationnel qui ne nie pas les substances mais les ignore en se refusant à les hypostasier. Il y a des hommes qui jouent des rôles. Il n'y a pas une Justice immuable et éternelle; il y a des homme qui disent la justice. Qu'ils le fassent en leur seul nom ou au nom d'un Dieu ne change rien à la représentation de la justice sur la scène : elle n'est pas cette vertu qui fait rendre à chacun son dû et redresse les torts de ceux qui en ont subi, conformément aux exigences d'un ordre chrétien authentifié ailleurs que sur la scène. Dans le théâtre tragique de Shakespeare, cet ordre n'est pas nié; il est absent $\mathrm{du}$ moins le plus souvent. La justice est présente, ô combien, mais comme construction d'hommes. Tout n'est que langage sur une scène qui n'affirme pas la consistance extra-textuelle d'un absolu : ce qui est juste, c'est ce qui est dit tel par celui qu'on ne peut contester. Rendre la justice, c'est la dire. Elle est aussi variée et multiple que la parole des hommes. Ce qui triomphe dans le théâtre de Shakespeare ce n'est pas quelque vue sublime de la justice divine ou d'un ordre naturel autour desquels les éléments d'une action seraient organisés comme chez Ben Jonson, ce sont des hommes qui se font leur justice en l'affirmant et en l'imposant.

L'absolu est absent parce qu'il est relativisé. Il n'existe que dans le discours des personnages et c'est la multiplicité même de sa proclamation qui le nie. Shakespeare n'est pas l'apologiste ou l'illustrateur d'un ordre juste, il est le re-présentateur de conduites humaines qu'il construit multiformes et contradictoires comme le réel dont il s'inspire. Tout se réduit à leur affrontement et à la résolution de ce conflit.

L'examen de quelques-unes des actions tragiques révèle l'inanité de la thèse d'un théâtre juste ou moral. Si la mort est le châtiment suprême de l'injustice, il s'en faut qu'elle ne frappe que les coupables. L'action tragique se 
caractérise par l'alternance de la violence qui passe d'un antagoniste à l'autre en un enchaînement que proposent la plupart des structures tragiques de Shakespeare. Titus Andronicus en apparait comme la première réalisation. Richard III présente une variation encore plus symétrique de cette alternance : les meurtres s'y répondent en une succession en soi interminable. Hamlet ne s'écarte pas du schéma fondamental : à la violence de Claudius, le héros tragique réplique par une autre violence qui gagne par contagion les proches du roi puis l'affecte à son tour. Cette alternance se trouve dans la plupart des tragédies' sinon dans toutes, mais elle n'est pas toujours celle de la rétribution, encore moins celle de la justice : la mort d'Ophélie ou même celle de Polonius ne peuvent se lire comme des manifestations de la justice. Il n'y a pas non plus de justice dans Romeo and Juliet : les violents des deux familles ennemies échappent même à toute rétribution et ce sont les non-violents qui meurent. L'injustice du dénouement est d'autant plus remarquable que nous savons que Shakespeare aurait pu le construire en conformité avec un schéma chrétien traditionnel. Il lui suffisait de suivre celui que lui proposait l'History'e of Romeus and Juliet de A. Brooke, source directe de la tragédie. Brooke avait donné à son histoire la forme morale d'une tragédie médiévale : Roméo et Juliet, en proie à une passion coupable, périssaient comme ils le méritaient, victimes malheureuses de la luxure :

"And to this end, good Reader, is this tragicall+ matter written, to describe unto thee a couple of unfortunate lovers, thralling themselves to unhonest desire, neglecting the authoritie and advice of parents and frendes, conferring their principall counsels with dronken gossyppes and superstitious friars, attemptyng all adventures of peryll for thattaynyng of their wished lust....»

Il est vain de se demander pour quelles raisons Shakespeare a modifié aussi radicalement sa source. Ce qui est évident, c'est qu'il écarte comme non tragique le schéma juste et moral. Ses amants ne meurent pas parce qu'ils sont coupables. C'est donc bien l'absence d'une référence à la justice et à un ordre moral qui est ici encore signifiante.

Le châtiment qui frappe le roi Lear et plus encore la mort scandaleusement injuste de Cordelia n'ont rien à voir 
avec la rétribution et sont même construits par Shakespeare en disproportion calculée par rapport à quelque faute intellectuelle ou morale qu'ils auraient pu commettre. Les références de certains personnages à la justice ne font qu'attirer l'attention du public sur cette incohérence : Albany se convainc aisément que les dieux ont puni Cornwall(IV.ii. 78-80) puis Goneril et Regan (V.iii.231-232), mais l'auteur lui fait alors constater que les dieux n'ont pas traité Cordelia avec la même justice et que tout son système s'effondre : «Albany, écrit Jacquot, se trouve (ainsi) placé dans une posture qui confine au ridicule ... S'il est une certitude (dans la pièce) et elle est négative - c'est qu'il n'existe pas de proportion entre la conduite morale des hommes et le sort qui leur est réservé» ${ }^{3}$.

La justice du nouveau roi dans Richard II n'est ni cohérente ni juste. Il pardonne à Aumerle à cause de l'intercession de sa mère, mais il fait périr ceux qu'il appelle des traîtres et qui n'ont eu que le tort de rester fidèles au roi vaincu. Il échappe lui-même - et c'est encore plus significatif dans la construction de la tragédie - aux conséquences morales du meurtre de Richard qu'il fait commettre par procuration. Au terme de la tragédie, le vrai meurtrier n'est pas jugé puisqu'il est le juge et que c'est lui qui définit la justice.

Il serait inutilement facile de continuer l'analyse des actions tragiques chez Shakespeare pour montrer qu'elles ne révèlent aucune cohérence du point de vue de la justice, aucune unité idéologique. La référence à la justice y est aussi constante que contradictoire : il n'y a pas une justice absolue en relation avec une vision morale ou métaphysique indiscutée qui, de l'extérieur, organiserait les pièces en leur imposant un modèle. Il y a autant de justices qu'il y a de voix qui triomphent au dénouement. C'est donc sur d'autres bases qu'il faut entreprendre l'examen des tragédies pour tenter d'y déceler un invariant qui n'est manifestement pas l'absolu d'une justice transcendante.

L'élément commun à toutes les tragédies de Shakespeare n'est pas la justice mais la violence. Et ceci, je l'espère, n'apparaitra pas comme une simple tautologie. Dans tous les cas sans exception, c'est par l'expulsion de la violence que la tragédie trouve son terme. Elle est exclue de la communauté qui proclame, souvent explicitement, que l'avenir sera 
non-violent. Il n'y a pas avènement d'une société juste : elle est fondée dans certains cas manifestement construits tels sur l'injustice préalable faite à certains individus : c'est indéniable dans Richard II, Romeo and Juliet, King Lear ou Timon of Athens. Ce n'est pas la justice qui est instaurée; c'est la violence qui est rejetée. Tous les dénouements tragiques de Shakespeare présentent une cohérence qui nª rien à voir avec la justice. Un examen rapide de quelques-uns de ces dénouements me paraît devoir apporter l'une des clefs du système tragique de Shakespeare. Ce qui donne en effet son sens à une histoire, c'est la fin et ce ne peut être que la fin. La forme d'un récit n'est perçue que lorsque la continuité des éléments narratifs retenus est interrompue par la discontinuité d'un dénouement qui opère sa clôture. La disposition des éléments narratifs est appréhendée dans la succession linéaire des épisodes, mais elle n'est perçue comme signifiante que dans sa totalité close. Comme tout poème - et toute histoire est poème, c'est-à-dire construction - le récit exige une lecture tabulaire, globale : c'est la fin qui lui donne son sens ${ }^{4}$. Il n'y a de perception que dans la différence. Or si on considère que c'est l'intertexte de Shakespeare qui constitue la norme, on ne peut que constater que les dénouements tragiques de notre dramaturge sont le plus souvent construits comme écarts par rapport à cette norme : le théâtre médiéval et son dérivé liumaniste chrétien de la Renaissance proposent toujours une fin morale alors que la fin tragique chez Shakespeare satisfait rarement cette exigence. C'est cet écart par rapport à une norme qui devient signifiance. Il oblige à chercher ailleurs l'invariant tragique de Shakespeare.

Cet invariant n'est pas simplement la non-violence proclamée au dénouement; il est aussi l'uniformité de son énonciation : dans tous les cas, le mot non-violent de la fin appartient à un violent ou - ce qui revient au même - au détenteur de la violence : prince, puissant, général victorieux. Il n'y a pas, je pense, d'exceptions dans le théâtre tragique de Shakespeare.

Dans Titus Andronicus, Lucius tue Saturninus, condamne Aaron à une mort horrible et annonce alors, et alors seulement, la fin de la violence :

«Then, afterward, to order well the state, That like events may never it ruinate.» 
Dans Richard II, Richard III, Macbeth et Julius Caesar, c'est le vainqueur qui instaure le nouvel ordre. C'est encore un guerrier victorieux qui clot Hamlet et Timon. Dans King Lear, c'est Edgar qui, après avoir tué Edmund, permet à Albany - un autre général victorieux - d'annoncer la fin de la violence :

«... We that are young

Shall never see so much nor live so long.»

Dans Othello et dans Romeo and Juliet, c'est un prince qui rétablit l'ordre parce que c'est à lui qu'il appartient d'imposer la non-violence. Lear a déchaîné la violence sans parvenir à la contrôler. C'est un violent victorieux qui y parviendra. Il diffère en cela du Bolingbroke de Richard II qui déchaîne lui aussi la violence mais qui l'organise victorieusement à son profit et qui la codifie dans un nouvel ordre proclamé non-violent. C'est accessoirement que la fin de la tragédie peut aussi marquer le triomphe de la justice comme dans Richard III ou Macbeth, mais ce n'est pas cette juste fin qui est significative puisqu'elle est le plus souvent absente ou volontairement obscurcie.

La tragédie de Brutus n'est pas qu'il tue César, c'est même son premier titre de gloire et celui qu'il revendique : «Stoop, Romans, stoop.

And let us bathe our hands in Caesar's blood Up to the elbows, and besmear our swords. Then walk we forth, even to the market place, And waving! our red weapons o'er our heads, Let's all cry : «Peace, freedom and liberty». »

(III.i. 106-111)

Cet épisode, s'il constituait la fin de la tragédie, pourrait apparaître comme un dénouement parfait : glorification de la violence libératrice associée à la proclamation de la fin de la violence. L'erreur tragique de Brutus, c'est d'affirmer sans réaliser : c'est ironiquement parce qu'il ne tue pas Antoine qu'il ne triomphe pas de la violence. C'est donc un autre qui aura le mot de la fin et non Brutus : un cynique que Shakespeare construit comme tel en le représentant dépourvu de scrupules moraux. L'ordre est rétabli injustement mais efficacement par Antoine et Octave; c'est bien leur injuste victoire qui instaure la non-violence du dénouement.

Tout se passe comme si le contenu juste ou injuste 
était in-signifiant dans ce système tragique si différent de la tragédie médiévale : on ne peut que constater une isomorphie remarquable de tous les dénouements : négation violente de la violence.

Mais il faut pousser plus loin l'analyse: ce rejet, cette expulsion de la violence passent toujours par l'inévitable mort d'une ou de plusieurs victimes sacrifiées pour que l'ordre non-violent puisse être instauré. Tous les dénouements tragiques marquent en effet un lien significatif entre la mort de la victime et l'instauration de l'ordre non-violent. La fin de Romeo and Juliet est particulièrement caractéristique mais elle n'est pas une exception : c'est parce que les deux amants meurent que les deux familles sont réconciliées. Parler d'isolement de la victime tragique dans la mort, ce n'est donc pas rendre compte du dénouement ou c'est n'en retenir qu'un aspect négatif. Le dénouement tragique opère la réunion de la communauté et réaffirme sa cohésion tout aussi explicitement que le dénóuement comique, quoique par d'autres moyens. La comédie se termine par la célébration d'un mariage; la tragédie se clôt par la célébration d'une mort violente qui met un terme à la violence. Pas plus qu'il n'est important de se demander si les protagonistes de la comédie méritent le dénouement heureux d'un mariage et d'un pardon, il n'est signifiant de porter un jugement de valeur sur la qualité morale de la victime. Son rôle structurel est de mourir pour expulser la violence. Et tout comme dans la comédie, il y a disproportion entre la cause et l'effet, entre l'erreur et le dénouement heureux, il y a disproportion dans la tragédie, mais dans l'autre sens : il n'y a pas adéquation entre la cause de la crise tragique et le dénouement violent. La fïn de la comédie comme celle de la tragédie est la célébration de l'ordre non la manifestation de la justice. Shakespeare termine ses comédies par un mariage et ses tragédies par une mort violente; dans les deux cas, le mot de la fin est unique : c'est la restauration de la paix. ${ }^{5}$

La seule fonction de la victime tragique est l'expulsion de la violence. Comme l'écrit René Girard à qui je me référerai souvent, «les tragiques nous montrent des personnages aux prises avec une mécanique de la violence dont le fonctionnement est trop implacable pour donner prise au moindre jugement de valeur, pour permettre toute distinction simpliste ou subtile entre les 'bons' et les 'méchants'. $\gg^{6}$. Pour lire le dénouement de la crise tragique, 
il convient en effet de remonter à son origine : la tragédie s'ouvre par l'instauration d'une violence qui est celle de frères ennemis et de doubles, écrit Girard; elle implique le refus de la différence culturelle, fondement de l'inégalité et de la hiérarchie : rivalité de Brutus et de César, de Claudius et d'Hamlet, d'Edgar et d'Edmund, de Macbeth et de Duncan, d'Othello et de Iago. Cette rivalité meurtrière engendre un processus de violence et de vengeance qui menace l'existence même du groupe. La résolution tragique rétablit les différences en séparant arbitrairement une victime qui porte le poids de la violence réciproque et dissimule au groupe la vérité de sa violence.

Bien des actions tragiques chez Shakespeare apparaissent comme l'illustration de cette lecture. Celle de Coriolanus est particulièrement probante. Non seulement le héros est instauré en étranger qu'on pourra commodément expulser, il veut l'être. Coriolanus se veut différent et la foule n'a plus qu'à le sacrifier puisqu'il l'est. Il se sépare et on le sépare. La scène du jugement populaire (III.iij) est l'essence même du processus de résolution de la crise tragique. A Sicinius qui lui dit :

«I do demand

If you submit you to the people's voices,

Allow their officers, and are content

To suffer lawful censure for such faults

As shall be proved unto you» (III.iii.43-47)

Coriolanus répond : «I am content»; et les tribuns le condamnent à l'exil «as enemy to the people and his country» (III.iii.118).Toute la foule reprend en choeur : «It shall be so». L'ennemi est expulsé et la communauté retrouve sa force : «Say their great enemy is gone, and they stand in their ancient strength» (IV.ii.6-7). Il mourra comme un traitre, mais la même communauté est prête à le glorifier après sa mort, qui est explicitement rattachée au déchaînement antérieur de la violence aussi bien qu'à son terme présent et à la certitude d'un avenir paisible :

«When you shall know the great danger

Which this man's life did owe you, you'll rejoice

That he is thus cut off» (V.vi. 137-140)

s'écrie Aufidius, qui poursuit dans un illogisme parfaitement conforme à la logique de la crise sacrificielle : 
Hath widowed and unchilded many a one

Which to this hour bawail the injury,

Yet he shall have a noble memory» (V.vi.152-155).

La violence qui est négation de la différence puis rétablissement violent de la différence apparaît aussi clairement dans Othello :

«That I did love the Moor to live with him, Thy downright violence and storm of fortunes May trumpet to the world.» (I.iii. 245-246)

déclare Desdomona. Une Vénitienne épouse un étranger, un barbare, niant la différence. Le duc de Venise reconnaît que c'est un désordre : «a mischief» (I.iii. 202). Othello accorde de l'avancement à Cassio sans tenir compte de l'ordre : «Preferment goes by letter and affection

And not by old gradation, where each second Stood heir to th'first» (I.i. 36-38), constate Iago. Ainsi, dès les premières scènes de la tragédie, la double victime est déjà séparée parce qu'elle a nié la différence. L'ordre sera rétabli avec son élimination violente. Ainsi la tragédie dissimule la vérité de la violence|rréciproque; elle déguise la crise sacrificielle en faisant de l'histoire tragiquel celle d'un homme, non celle du groupe.

Mais Shakespeare représente trop fidèlement la vérité des antagonismes des doubles pour tomber dans un moralisme facile, sauf peut-être dans Richard III. Son système tragique est trop subtil pour se laisser aller aux aisances dangereuses du mélodrame ou de la pièce à thèse.Sa tragédie exige des antagonistes symétriques, des doubles presque parfaits : Capulet et Montague, Brutus et César, Antoine et Cléopâtre : la violence est réciproque puisqu'elle est celle des doubles; il n'y a pas d'un côté un bon et de l'autre un méchant. Même dans Richard III, surtout dans Richard III. la violence est celle des doubles : un Richard correspond à un autre Richard; un Edouard est mis en parallèle avec un autre Edouard ... Ils sont frères dans la violence, mais la tragédie isolera la victime qui mettra fin au cercle infernal de la vengeance.

«Le mécanisme de la violence émissaire, écrit toujours Girard, est doublement salvateur; en réalisant l'unanimité, il fait taire la violence sur tous les plans où elle parle; il empêche les proches de se battre et il empêche la vérité de l'hom- 
me d'apparaître, il la pose à l'extérieur de l'homme comme incompréhensible divinité $\gg{ }^{7}$.

Le groupe se décharge de sa violence sur une victime qui ne remplit son rôle que parce qu'elle est incapable de provoquer des représailles. Le dénouement tragique interrompt définitivement le processus de violence réciproque, l'enchaînement de la vengeance. c'est ainsi que l'incohérence même des actions tragiques devient le signe de leur sens. C'est encore et toujours la forme qui est signifiante et non son contenu : la tragédie, c'est la fin violente de la violence, quelle que soit sa matière, quel que soit le statut moral de son héros.

Comme les grands tragiques grecs, Shakespeare montre l'homme aux prises avec ce que Girard appelle la mécanique de la violence qui confond bons et méchants dans l'indifférenciation violente des doubles :

«Les préférences affichées dans un sens ou dans l'autres n'empêchent jamais les auteurs tragiques de souligner à chaque instant la symétrie de tous les antogonismes» ${ }^{8}$.

C'est le rationalisme classique qui tentera de séparer les bons et les méchants dans sa vaine entreprise de moralisation de la tragédie shakespearienne. Shakespeare ne fait rien de tel et, pour sortir de la violence, le dénouement de ses tragédies ne recourt pas à l'idée de rétribution ou de justice. Il n'y a peut-être pas de tragédie sans injustice et sans scandale. Même la tragédie médiévale parvenait, par d'autres moyens que ceux des Grecs ou de Shakespeare, à opérer l'indifférenciation des antagonismes, prélude nécessaire à la sélection d'une victime : tous les hommes étant pécheurs étaient par définition coupables et tout châtiment était donc mérité, même s'il ne frappait arbitrairement que certaines victimes exemplaires.

Les tragédies de Shakespeare ne se terminent donc pas par l'apothéose de la justice, sauf s'il faut appeler justice la violence du plus fort. C'est parce que celui qui met un terme violent à l'enchaînement de la violence réciproque n'a pas à craindre de devenir à son tour victime qu'il appelle juste l'ordre qu'il instaure. Il n'y a pas de différence de nature entre vengeance et justice. Toutes deux sont violentes et punissent le crime. La première est un processus interminable; la seconde interrompt violem- 
ment le processus pour instaurer la non-violence. «La justice c'est toujours le dernier mot de la vengeance» ${ }^{9}$.

Le théâtre de Shakespeare peut donc être lu non pas tellement comme la négation de toute justice, mais au contraire comme un modèle de son fonctionnement : il n'y a pas de bonne ou de mauvaise justice, il n'y a que la justice qui remplit son rôle qui est d'empêcher le retour de la violence anarchique. La "vérité» de la justice dans un système culturel, c'est son aptitude à assurer la paix. Ce que le jardinier reproche à Richard II, ce n'est pas d'avoir été injuste, c'est de ne pas avoir jugé et donc régné. Bolingbroke est un bon roi puisqu'il juge et impose son ordre, et cet ordre est juste puisqu'il met fin à la violence, tout comme dans Richard III, Hamlet ou Macbeth. Ce qui importe pour la résolution de la crise tragique, c'est qu'une victime meure, devenue impuissante et donc bouc émissaire, pour que l'ordre revienne et que la violence soit exorcisée. Le théâtre de Shakespeare n'est jamais plus humain que lorsqu'il sacralise dans la violence le terme de la violence.

«Au cercle vicieux de la violence réciproque totalement destructrice se substitue ... le cercle vicieux de la violence rituelle créatrice et protectrice ... La violence devient le signifiant du désirable absolu, de l'auto-suffisance divine. Le sujet adore cette violence et il la hait; il cherche à la maîtriser par la violence...»" C'est Macduff qui, la tête de Macbeth à la main, proclame l'avènement d'un nouvel ordre, tout comme Richmond sur le cadavre de Richard, ou comme Octave près de celui de Brutus, ou comme Edgar terrassant Edmund. La tragédie ne met un terme à la violence qu'en sacralisant le violent et en le séparant à la fois.

Si le théatre de Shakespeare reste actuel, c'est qu'il échappe aux limites d'une idéologie, d'une morale et d'une justice pour retrouver la vérité des relations humaines sous le masque divers des cultures. La tragédie de Shakespeare est immortelle parce qu'elle célèbre le rite fondateur de toute culture : l'expulsion de la violence réciproque paradoxalement opérée dans un meurtre rituel. Ordre et désordre s'y opposent non pas comme justice à injustice mais comme violence sacralisée à violence anarchique. Le rôle du rite d'expulsion n'est pas signifiant par son contenu moral ou juridique mais par sa forme seule : rejet violent d'une vio- 
lence. Les héros tragiques sont tour à tour et parfois simultanément - Hamlet en est une admirable illustration oppresseurs et opprimés, bourreaux et victimes, méchants et bons, vengeurs et objets de vengeance. La tragédie renverse les rôles : le roi devient le non-roi, et c'est bien ce caractère énigmatique et cette incohérence qui fondent la tragédie. Toute simplification manichéenne qui opérerait dès le début de la crise tragique la séparation des bons d'avec les méchants évacuerait le tragique, même et surtout si elle était entièrement conforme à la justice et à la morale. Le tragique, c'est la violence; elle n'est ni bonne ni mauvaise, elle est.

Shakespeare n'est pas juste et c'est pour cette raison qu'il dure. Son théâtre n'est pas engagé parce que l'engagement, c'est encore et toujours la sacralisation d'un ordre et d'une idéologie particuliers. Ce que les tragédies de Shakespeare représentent, ce n'est pas la «vérité» d'une justice particulière mais la robuste vitalité de toute culture qui définit son espace juridique en le fondant sur la violence pour pouvoir la nier. La tragédie célèbre autant la vie que ne le fait la comédie. Il n'est pas possible de voir, contrairement à ce que propose N. Frye, dans cette dernière la célébration des valeurs collectives de vie, symbolisées par l'avènement d'une nouvelle société, et dans la première une sorte de rite d'exclusion d'un individu, solitaire devant la mort ${ }^{11}$. L'une et l'autre réconfortent le groupe et ouvrent les mêmes perspectives d'ordre et de non-violence. L'une et l'autre finissent bien puisqu'elles mettent un terme au conflit. Seuls les moyens diffèrent.

La tragédie de Shakespeare est ouverte, interrogative. Elle n'apporte pas une réponse idéologique à toutes les situations tragiques. Elles représente les hommes aux prises avec leur violence et s'en libérant, parfois dans un contexte chrétien, parfois et même plus souvent hors de toute référence à la philosophie et à la métaphysique du temps. Et c'est peut-être parce que ce modèle tragique est élaboré à l'écart de la littérature qu'il y parvient : il ne se contruit pas en référence aux conventions de ce discours à l'époque élisabéthaine. Shakespeare se contente de proposer aux spectateurs ce qui les incitera à revenir dans son théâtre. Il ne cherche pas à les rendre meilleurs mais à les satisfaire.

Il n'y parvient sans doute que parce que son théâtre représente un moment fugitif dans l'évolution de la culture 
occidentale, caractérisé, sur la scène populaire de la fin du règne d'Elizabeth, par l'absence tolérée d'une norme idéologique stricte dans des spectacles qui ne sont pas condamnés à être édifiants. Plusieurs décennies avant la fermeture des théâtres en 1642 , cette heureuse absence disparaîtra et les héritiers et successeurs de Shakespeare se verront à nouveau enfermés dans des formes dramatiques qui les obligeront à séparer le bien du mal, évacuant peut-être ainsi l'essence même du tragique. Car la tragédie n'est pas l'épiphanie de la justice : «la tragédie est l'équilibre d'une balance qui n'est pas celle de la justice mais de la violence. ${ }^{12}$

Pierre SPRIET

\section{NOTES}

1. Cf.J. Danby, Elizabethan and Jacobean Poets, London : Faber \& Faber, 1955,pp. 71-73.

2. G. Bullough, Narrative and Dramatic Sources of Shakespeare, I, London, 1966, p. 284.

3. Les Voies de la Création Théâtrale, VI (1978), p. 489.

4. Cf. F. Kermode, The Sense of an Ending.

5. Comme le note $B$. Beckerman, toutes les pièces de la grande période du Globe se terminent par ce qu'il appelle une "public resolution» : Shakespeare at the Globe, 1599-1609, New York: MacMillan, 1962, p. 39.

6. R. Girard, La Violence et le sacré; Paris : Grasset, 1972, p. 74.

7. Ibid., p.382.

8. Ibid., p.72

9. Ibid., p.32.

10. Ibid., p. 208.

11. Ibid., p. 72 .

12. Ibid.,p. 72 . 